\title{
Discriminação fonológica e memória em crianças com dislexia e bons leitores.
}

\author{
Katerina Lukasova - Universidade Cruzeiro do Sul, São Paulo, Brasil \\ Anna Carolina Cassiano Barbosa - Universidade Presbiteriana Mackenzie, São Paulo, Brasil \\ Elizen Coutinho de Macedo - Universidade Presbiteriana Mackenzie, São Paulo, Brasil
}

\begin{abstract}
Resumo
Presente estudo avaliou a capacidade de discriminação fonológica de sílabas em crianças com dislexia do desenvolvimento e bons leitores. Participaram do estudo 10 disléxicos e 10 controles pareados por idade, sexo, série, nível intelectual e atencional. 45 pares de sílabas diferentes quanto à sonorização, ponto de articulação e sílabas iguais foram apresentados para julgamento de similaridade em provas SOM/SOM e SOM/ESCRITA. O intervalo entre estímulos variou de curto, médio e longo. Os resultados mostraram diferenças entre-grupo em tarefa SOM/SOM, sendo que os disléxicos obtiveram desempenho inferior aos bons leitores em sílabas sonoras e ponto de articulação com intervalos longos. Análise dos pares de sílabas mostrou baixo acerto dos disléxicos nas sonoras (/KA/-/GA/ e /FA/-/VA/) e ponto de articulação (/ZA/-/VA/). Os resultados mostram perda mais rápida da informação fonológica na memória de trabalho em crianças disléxicas.

Palavras-chave: dislexia; discriminação fonológica; memória de trabalho.
\end{abstract}

\section{Phonological discrimination and memory in children with dyslexia and good readers}

\begin{abstract}
The present study assessed the syllables' phonological discrimination ability in children with developmental dyslexia and good readers. 10 dyslexics and 10 controls matched for age, gender, school grade, intelligence and attention participated in the study. 45 pairs of syllables differentiated by sound, point of articulation and identical syllables were presented in forced choice tests SOUND/SOUND and SOUND/LETTER. The inter stimuli time varied from short, medium and long. The results showed between-group differences in SOUND/SOUND test with dyslexics doing worse than good readers in sound syllables and point of articulation syllables with long intervals. Analysis of the syllables pairs showed lower score for dyslexics in sound syllables (/KA/-/GA/ and /FA/-/VA/) and point of articulation syllables (/ZA/-/VA/). The results showed faster loss of phonological information in dyslexic children working memory.

Keywords: dyslexia; phonological discrimination; working memory.
\end{abstract}

\section{Introdução}

A dislexia do desenvolvimento é, de acordo com a Associação Internacional de Dislexia, um transtorno específico de aprendizagem, caracterizado pela dificuldade na correta e/ou fluente leitura de palavras, na escrita e nas habilidades de decodificação. Estas dificuldades são tipicamente decorrentes de um déficit no componente fonológico da linguagem que freqüentemente não é esperado em relação a outras habilidades cognitivas e à provisão de adequada instrução escolar. As conseqüências destas dificuldades podem incluir problemas na compreensão da leitura e diminuição das oportunidades de leitura pelo fato da criança evitar entrar em contato com material escrito. Tais problemas causam grande impacto na atividade

\footnotetext{
${ }^{1}$ Endereço para correspondência:

Laboratório de Distúrbios do Desenvolvimento, Prédio Amantino Vassão (térreo), Rua da Consolação, 896; Consolação, 01302-907; São Paulo, S.P. Email: katerinaluka@gmail.com, elizeumacedo@uol.com.br.

Agradecemos a colaboração de Yvan Leonardo Barbosa Lima na gravação dos testes.
}

acadêmica pois impedem, por exemplo, ampliação do vocabulário e obtenção de conhecimento geral (Lyon, 2003).

A dislexia do desenvolvimento não pode ser considerada um distúrbio homogêneo, pois apresenta uma gama ampla de sinais comportamentais e cognitivos. Um dos sinais cognitivos observados é a dificuldade de discriminar sons em seus aspectos fônicos ou temporais. Bishop e colaboradores (1999) identificaram, em crianças disléxicas, baixa habilidade de reconhecer informações acústicas em alternância rápida, tais como a identificação e o seqüenciamento de sons diferentes, como as sílabas / ba/ e /da/ apresentadas em intervalo de tempo variável.

Para comparar e distinguir dois sons distintos, diferentes aspectos acústicos e articulatórios devem ser analisados, entre tais aspectos destaca-se o "voice-onset time" (VOT). O VOT é definido como o espaço de tempo entre o início da vibração das cordas vocais e abertura da boca para a vocalização do som. Assim, diferentes fonemas apresentam diferentes VOTs. Os fonemas bilabiais, /b/ e /p/, são produzidos pela abertura 
brusca de lábios, mas apresentam VOTs distintos. O início da vocalização do fonema /p/ se dá cerca de 40 a 60 milésimos de segundos após o fonema /b/. A fim de verificar a capacidade de discriminação em disléxicos e controles, Ackermann \& Hertrich (1997) manipularam a duração do VOT em duas palavras alemãs /boden/ e /boten/. Entre a vocalização de duas sílabas da palavra/bo-den/, acontece uma breve oclusão de ar com duração de 10 milésimos de segundos. Com a extensão sucessiva desse período por mais 10 milésimos de segundos, foi criado um espectro de palavras intermediárias entre palavras alvo /boden - boten/. Adultos com dislexia obtiveram desempenho inferior aos controles na diferenciação das palavras intermediárias dos alvos (Serniclaes \& cols, 2004a). O estudo foi repetido com adultos analfabetos, a fim de excluir a possibilidade de que o déficit seja causado pelo pouco contato material escrito. Os resultados mostraram que os adultos analfabetos e os controles apresentaram o mesmo padrão de discriminação, ou seja, a falta de treino em leitura não leva ao déficit na percepção sonora, ao contrário, o déficit da discriminação é que pode ter um efeito negativo no desenvolvimento de habilidades de leitura e escrita em disléxicos.

Repovs e Baddeley (2006) indicam que a memória fonológica de trabalho envolve a reverberação do traço acústico da informação falada por alguns segundos no buffer fonológico e na alça fonológica onde é reverberada por meio da repetição sub-vocal. Assim, uma seqüência de sons dissimilares é mais facilmente memorizada, enquanto que as seqüências similares são mais difíceis. Estas dificuldades podem ser explicadas pelo fato das seqüências similares sofrerem interferência dos próprios traços sonoros ocasionando diminuição do número de sons evocados corretamente (Repovs \& Baddeley, 2006). Kibby e colaboradores (2004) avaliaram crianças com dificuldades de leitura em tarefas de reverberação e armazenamento da informação fonológica. As crianças com dificuldades na leitura mostraram resultados inferiores aos bons leitores. A discrepância entre os dois grupos foi ainda maior quando o grau de complexidade da tarefa foi aumentado, exigindo-se ainda mais a reverberação fonológica. Não foram encontradas diferenças entre os dois grupos quando realizaram tarefas que envolviam apenas memória não verbal.

No Brasil, Capovilla e Capovilla (2002) avaliaram discriminação silábica em bons e maus leitores da primeira a quarta série. Pares de sílabas eram iguais ou diferentes quanto à sonorização, ponto de articulação, modo de articulação ou mistas. O tempo entre a aparição da primeira e segunda sílaba variou de 20 milésimos de segundos até 60 segundos. Os resultados indicaram proporção de acerto significativamente maior para os bons leitores sendo que em ambos os grupos, as sílabas com diferenças sonoras e ponto de articulação foram aquelas que os sujeitos mais erraram. Quanto a distribuição de acerto, em função do intervalo temporal entre as sílabas, a menor diferença na proporção de acerto foi observada nos intervalos de 2,5 segundos. O aumento ou diminuição do intervalo produziu redução no número de acerto para ambos os grupos, com os maus leitores pontuando menos que os bons leitires. Os maus leitores apresentaram dificuldades relacionadas com a velocidade de processamento e como a memória fonológica de trabalho.

O presente estudo se propõe a avaliar a capacidade de discriminação de sílabas pareadas por similaridade sonora e ponto de articulação em crianças disléxicas e bons leitores. Uma vez que os disléxicos e maus leitores apresentam déficits relacionados com o processamento fonológico e a memória fonológica a caracterização do padrão de respostas em provas que envolvam estas habilidades pode contribuir para o estabelecimento de diferentes perfis para esse grupo. Tal achado pode contribuir com o desenvolvimento de novas provas de avaliação com bom poder discriminativo e fácil aplicação computadorizada.

\section{Método}

\section{Sujeitos}

Inicialmente foram avaliadas 20 crianças com diagnóstico de dislexia. As crianças foram encaminhadas pelo Núcleo de Atendimento Neuropsicológico Infantil Interdisciplinar (NANIUNIFESP), e por CECOP, consultório particular de atendimento fonoaudiológico. A participação de todos os sujeitos foi voluntária com a assinatura dos pais ou responsáveis do Termo de Consentimento Livre e Esclarecido.

A fim de confirmar o diagnóstico e satisfazer os critérios de inclusão e exclusão, novas avaliações foram feitas em cada uma das crianças encaminhadas. Assim, todos os participantes foram avaliados por meio de uma bateria de testes psicológicos e de leitura e escrita, a fim de confirmar o diagnóstico de dislexia. Foram incluídas no estudo apenas crianças que 
apresentavam desempenho cognitivo bom, mas nível de leitura inferior ao esperado para a série escolar.

O nível cognitivo foi avaliado pela Escala de Inteligência Wechsler para Crianças (WISC-III; Wechsler \& Figueredo, 2002) sendo incluídos somente participantes com QI acima de percentil 25. O nível de leitura e escrita foi avaliado com testes da Bateria de Leitura e Escrita Computadorizada (BALE-Comp, Macedo \& cols., 2005). A bateria possibilita avaliação de competência e velocidade de leitura e escrita por meio de 4 testes: Teste de Competência de Leitura de Sentenças (TCLS), Teste de Nomeação de Figuras por Escrita (TNF 1.1-Escrita); Teste de Nomeação de Figuras por Escolha (TNF 1.1Escolha) e Teste de Competência de Leitura de Palavras (TCLP) no qual a criança deve julgar a ortografia de palavras isoladas. O baixo nível de desempenho e/ou velocidade de leitura e escrita foi considerado com a pontuação um desvio padrão abaixo da pontuação padrão encontrada em crianças leitores normais da mesma série escolar.

As crianças com histórico de comorbidades psiquiátricas, neurológicas ou evasão escolar foram excluídas. Não participaram crianças que obtiveram um desempenho abaixo de percentil 25 na prova de Atenção Concentrada (AC).

Ao final, das 20 crianças encaminhadas com o diagnóstico de dislexia, apenas 10 foram incluídas. O grupo controle foi formado por 10 crianças pareadas por idade, sexo e série. A fim de caracterizar os dois grupos, foram conduzidas análises estatísticas. Não foram observadas diferenças na pontuação em teste de inteligência geral e de atenção. No entanto, disléxicos apresentaram desempenho inferior ao controles na escrita (TNF 1.1-Escrita), julgamento ortográfico (TCLP), leitura com escolha de palavra adequada (TNF 1.1-Escolha) e maior tempo na leitura de sentenças (TCLS). A caracterização dos sujeitos está descrita na Tabela 1.

Tabela 1. Teste $t$ da média de acerto e/ou velocidade de execução em provas de caracterização cognitiva.

\begin{tabular}{lcccccc}
\hline & Disléxicos & & Controles & $\mathrm{t}(1,18)$ & $p$ \\
\hline & média & $\mathrm{dp}$ & média & $\mathrm{dp}$ & & \\
\hline Número de sujeitos & 10 & & 10 & & & \\
Meninos & 8 & & 8 & & & \\
Idade (anos) & 10,90 & 0,9 & 11,08 & 1,3 & 0,365 & 0,719 \\
Escolaridade (anos) & 4,80 & 1,13 & 4,80 & 1,39 & 0,000 & 1,000 \\
WISC (total) & 110,10 & 11,64 & 115,10 & 15,72 & 0,808 & 0,429 \\
AC (percentil) & 51,50 & 17,48 & 61,00 & 14,49 & 1,323 & 0,202 \\
TCLS (tempo) & 5,00 & 2,31 & 3,16 & 0,55 & 2,443 & $\mathbf{0 , 0 2 5}^{*}$ \\
TNF 1.1-Escrita (acerto) & 25,30 & 4,03 & 30,40 & 3,75 & 2,931 & $\mathbf{0 , 0 0 9}^{*}$ \\
TCLP (acerto) & 60,40 & 5,00 & 65,10 & 4,5 & 2,209 & $\mathbf{0 , 0 4 0}^{*}$ \\
TNF 1.1-Escolha (acerto) & 33,30 & 1,34 & 35,00 & 1,16 & 3,042 & $\mathbf{0 , 0 0 7}^{*}$ \\
\hline
\end{tabular}

${ }_{* \mathrm{p}}^{\mathrm{p}}<0,05$

\section{Instrumentos}

Foram aplicadas duas provas computadorizadas de discriminação de sílabas: SOM/LETRA e SOM/SOM. Na prova SOM/LETRA, o participante deveria julgar se o som de uma sílaba correspondia a uma sílaba escrita que aparecia na tela do computador. As sílabas eram escritas com letra minúscula em negrito, fonte Arial, tamanho 58. $\mathrm{Na}$ prova SOM/SOM, o participante deveria julgar se o som de uma sílaba correspondia ao de outra sílaba apresentada posteriormente. Os sons das sílabas eram apresentados em formato digitalizado de voz natural masculino, com sotaque da cidade de São Paulo.
Todas as sílabas eram compostas por uma consoante e a vogal "a". As consoantes foram classificadas quanto à sonorização (sílabas SS) ou ponto de articulação (sílabas SP). As consoantes compondo sílabas SS foram pareadas dentro do mesmo grupo articulatório, por exemplo, consoantes bilabiais, labiodentais, alveolares, palatais ou velares. Dessa maneira, os estímulos SS eram compostos por consoantes $/ \mathrm{p} /-/ \mathrm{b} /, / \mathrm{t} /-/ \mathrm{d}$, $/ \mathrm{f} /-/ \mathrm{v}, / \mathrm{s} /-/ \mathrm{z} / \mathrm{e} / \mathrm{k} /-/ \mathrm{g} /$. Os pares apresentados poderiam ser distintos apenas dentro do mesmo grupo articulatório do tipo SS: /PA/-/BA/; /TA/-/DA/; /FA/-/VA/;/SA/-/ZA/; /KA//GA/. Já as consoantes compondo sílabas SP foram pareadas entre os grupos articulatórios: /f/- 
/s/, /n/-/m/, /v/-/z/, /t/-/p/ e /b/-/d/. Os pares SP foram: /FA/-/FA/; /SA/-/SA/; /NA//NA/;/MA/-/MA/;/VA/-/VA/;/ZA/-/ZA/; $/ \mathrm{TA} /-/ \mathrm{TA} / ; / \mathrm{PA} /-/ \mathrm{PA} / ; / \mathrm{BA} /-/ \mathrm{BA} / ; / \mathrm{DA} /-$ $/ \mathrm{DA} /$. Os pares das sílabas iguais (SI) foram compostos por consoantes escolhidas entre as SS e $\mathrm{SP}$ e foram: /PA/-/PA/; /BA/-/BA/; /TA//TA/; /DA/-/DA/; /FA/-/FA/; /VA/-/VA/; /SA/-/SA/; /ZA/-/ZA/; /KA/-/KA/; /GA//GA/, /MA/-/MA/; /NA/-/NA/.

Assim, tanto a prova SOM/SOM, quanto a prova SOM/LETRA era formada por 45 pares de estímulos que os participantes deveriam avaliar. Destes, 15 pares eram diferentes entre si com relação à sonorização (SS); 15 eram distintos entre si, mas em função do ponto de articulação (SP); e 15 pares os estímulos eram idênticos (SI).

$O$ intervalo entre estímulos (IEE) foi definido como tempo entre o fim da vocalização da primeira sílaba e a apresentação da segunda sílaba do par. O IEE foi controlado para cada seqüência de sílabas em três grupos: curto, médio e longo. Os IEEs nos intervalos curtos foram: 50; 300; 600; 900; 1200 milésimos de segundos. Nos intervalos médios foram: 2000; 2800; 3600; 4400; 5200 milésimos de segundos. Já nos intervalos longos foram: $10 ; 20 ; 30 ; 40 ; 50$ segundos. Cada par de sílabas foi apresentado com uma duração intervalar fixa em cada seqüência, por exemplo, a sílaba /ba/-/da/ foi apresentada com intervalos de $50 \mathrm{~ms}, 2000 \mathrm{~ms}$ e 10 segundos. Durante os intervalos sonoros, em $1 / 3$ do tempo era apresentado um sinal sonoro que se caracterizava como uma interferência lingüística, composta por um conjunto de fragmentos silábicos incompreensíveis. Isto foi feito para dificultar a reverberação do estímulo apresentado primeiro. Tanto a ordem de apresentação das sílabas dentro de cada um dos pares, quanto a seqüencia de apresentações dos pares em cada teste foi aleatorizada.

\section{Procedimento}

A criança sentava à frente do computador e foi dada a instrução da tarefa. A fim de verificar se a criança havia compreendido a instrução, duas telas de treino foram apresentadas. No caso de erro, a instrução foi dada novamente. A duração média de cada teste foi de 20 minutos, por tanto os testes foram aplicados em sessões diferentes ou no começo, ou fim da sessão, evitando efeito de fadiga.

\section{Resultados}

Inicialmente foi avaliado o efeito da tarefa (SOM/SOM e SOM/LETRA) na média de acerto por meio da ANOVA multifatorial. Os disléxicos mostraram média de acerto inferior aos controles na tarefa SOM/SOM $(\mathrm{F}[1,18]=4,813 ; \mathrm{p}<0,042)$. Não foram observadas diferenças significativas na tarefa SOM/LETRA. As médias de acerto estão descritas na Tabela 2. As análises das variáveis, tipo de sílabas e IEEs, foram feitas separadamente para cada tarefa.

Tabela 2. Média de acerto para os disléxicos e controle por tarefa e tipo de sílaba.

\begin{tabular}{lllllll}
\hline Tarefa & Disléxicos & $\mathrm{dp}$ & Controle & $\mathrm{dp}$ & $F$ & $p$ \\
\hline SOM/SOM (1) & 76,44 & 10,79 & 84,89 & 05,62 & 4,813 & $\mathbf{0 , 0 4 2 ^ { * }}$ \\
Sílabas SS (1) & 62,66 & 21,59 & 72,66 & 14,89 & 1,206 & $\mathbf{0 , 2 4 4}$ \\
Sílabas SP (1) & 84,66 & 10,44 & 93,33 & 5,44 & 2,327 & $\mathbf{0 , 0 3 2}^{*}$ \\
Sílabas SI (1) & 82,00 & 12,59 & 88,66 & 7,73 & 1,147 & 0,171 \\
SOM/LETRA (2) & 79,33 & 13,90 & 83,11 & 13,58 & 0,378 & 0,546 \\
Sílabas SS (2) & 77,09 & 16,74 & 78,66 & 21,95 & 0,180 & 0,859 \\
Sílabas SP (2) & 82,33 & 14,28 & 90,00 & 7,85 & 1,599 & 0,127 \\
Sílabas SI (2) & 84,83 & 14,28 & 80,66 & 18,17 & 0,570 & 0,576 \\
\hline
\end{tabular}

$* \mathrm{p}<0,05$

\section{Tarefa $S O M / S O M$}

O efeito do tipo de sílaba (SP, SS e SI) na média de acerto dos grupos foi comparado por meio das análises do teste $t$ das amostras independentes. Apenas nas sílabas SP foi observada uma média de acerto significativamente inferior $(\mathrm{t}[1,18]=2,327 ; p<0,032)$ para o grupo dos disléxicos (média $=84,66 ; \quad \mathrm{dp}=10,44$ ) quando comparados com controles (média $=93,33 ; \mathrm{dp}=5,44)$. Os resultados estão descritos na Tabela 2 .

A fim de verificar o efeito da duração intervalar (curto, médio e longo) na média de acerto em cada tipo de sílabas (SS, SP e SI) foram novamente conduzidas análises com o teste $t$. A média de acertos para os disléxicos foi inferior 
Lukasova, K., Barbosa, A. C. C., Macedo, E. C. Discriminação fonológica em crianças disléxicas para a duração intervalar longa em sílabas SS $p<0,054)$. Os resultados estão descritos nas $(\mathrm{t}[1,18]=2,058 ; \quad p<0,054) \quad$ e $\mathrm{SP} \quad(\mathrm{t}[1,18]=2,058 ; \quad$ Figuras 1 à 3.

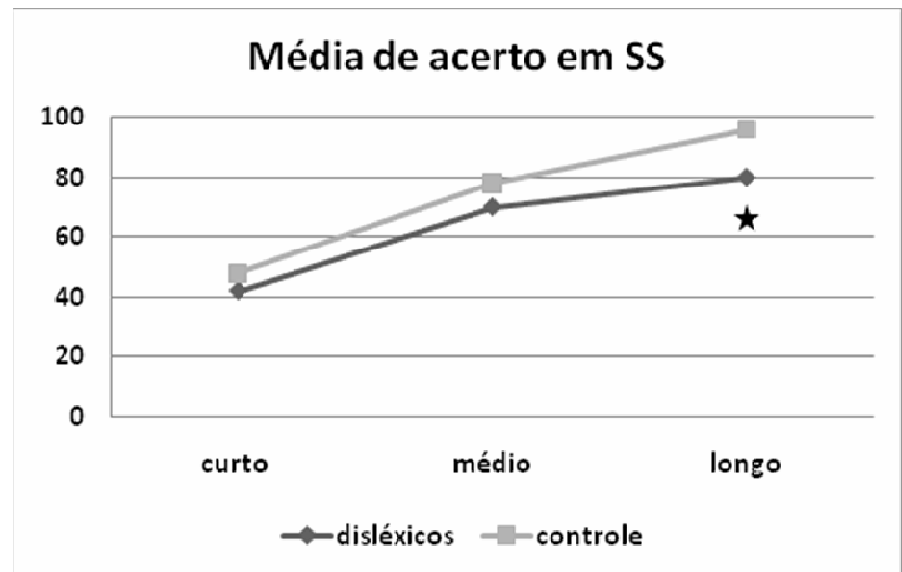

Figura 1. Média de acerto em função de tipo de sílaba SS $\left({ }^{*} p>0,5\right)$.

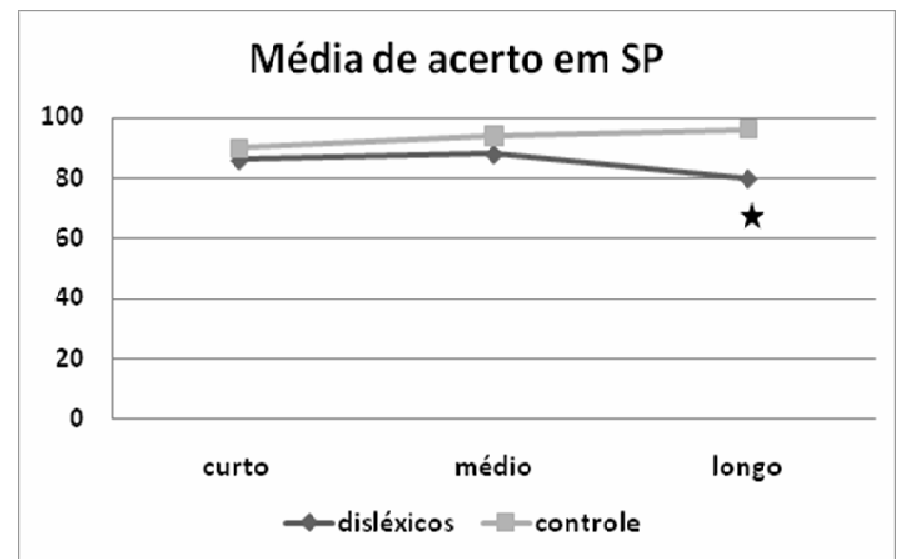

Figura 2. Média de acerto em função de tipo de sílaba SP $\left({ }^{*} p>0,5\right)$.

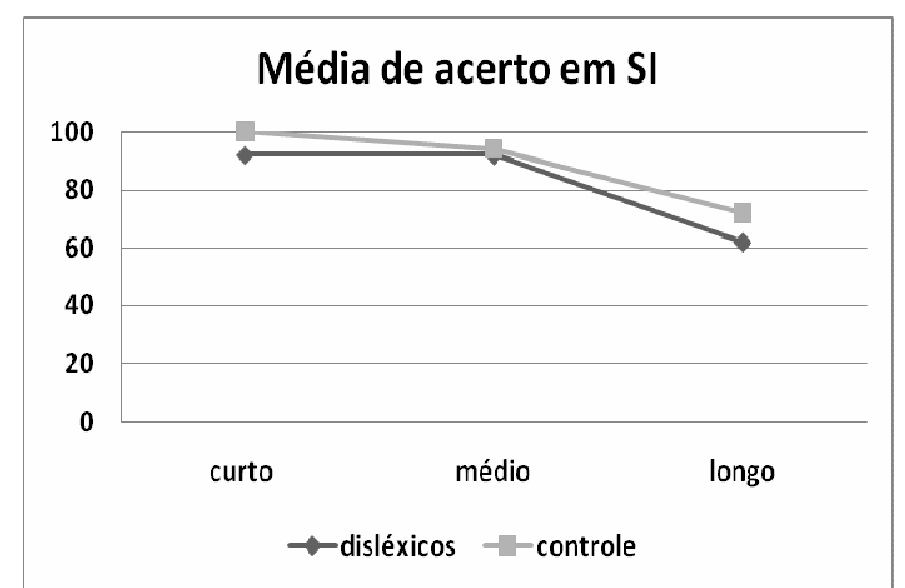

Figura 3. Média de acerto em função de tipo de sílaba SI.

O efeito dos pares de sílabas, compondo os grupos SS e SP, na média de acerto foi analisado com a prova de medidas repetidas na qual foram considerados os 3 tipos de intervalo: curto, médio e longo; e os 5 tipos de pares de sílabas. 
Em pares de sílabas $S S$ a análise mostrou efeito principal para sílaba $(\mathrm{F}[8,144]=2,190$; $p<0,031)$ e uma tendência para interação sílaba e grupo $(\mathrm{F}[4,72]=2,134 ; p<0,085)$. Teste $t$ mostrou pior desempenho para os disléxicos na discriminação das sílabas KA-GA (t[18]=2,278; $p<0,035)$ e FA-VA (t[18]=2,212; $p<0,046)$. Em relação à duração intervalar, o desempenho inferior dos disléxicos foi observado em tempo intervalar curto nas sílabas KA-GA (t[18]=2,058; $p<0,054)$ e longo nas sílaba FA-VA (t[18]=2,058; $p<0,054)$. Os valores estão descritos na Figura 4.

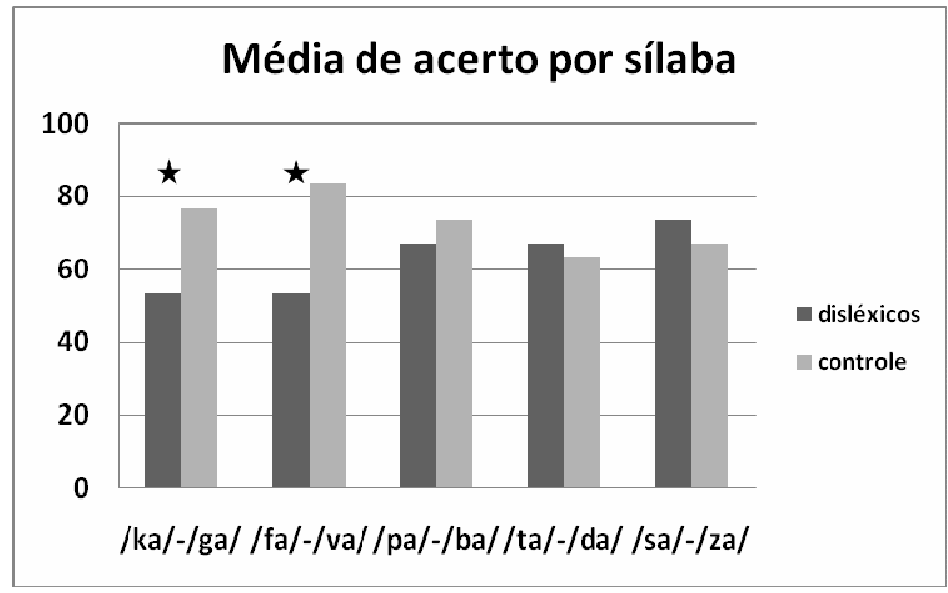

Figura 4. Média de acerto em função de pares de sílabas SS $\left({ }^{*} p>0,5\right)$.

Em pares de sílabas SP a análise mostrou efeito principal para sílaba $(\mathrm{F}[4,72]=4,683$; $p<0,002)$ e efeito intra grupo $(\mathrm{F}[1,18]=5,413$; $p<0,032)$. O teste $t$ de amostras independentes mostrou pontuação média inferior em grupos de disléxicos na discriminação de sílabas ZA-VA $(\mathrm{t}[18]=2,236 ; p<0,052)$. Os valores estão descritos na Figura 5.

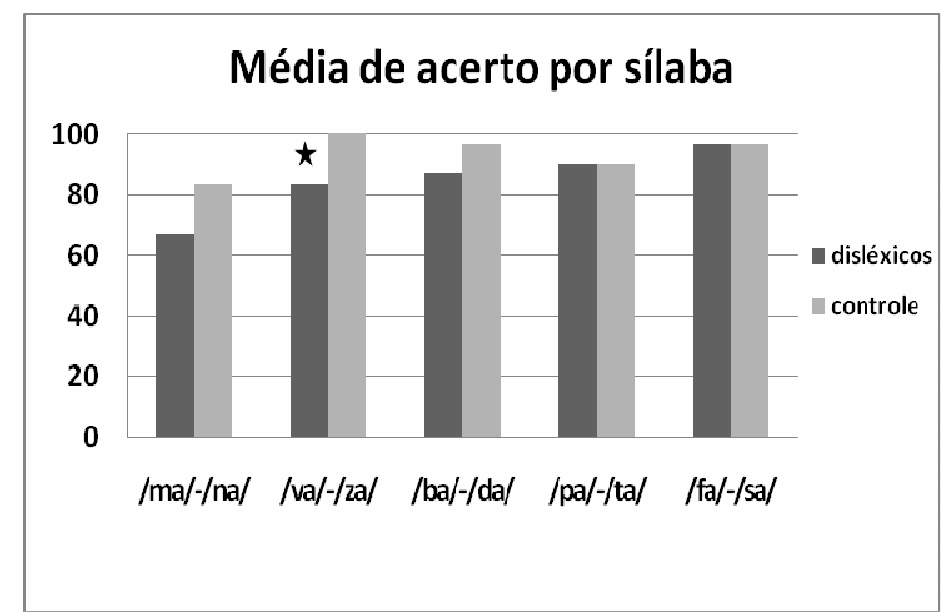

Figura 5. Média de acerto em função de pares de sílabas SP $\left({ }^{*} p>0,5\right)$.

Tarefa SOM/LETRA

O efeito do tipo de sílaba (SP, SS e SI) e tempo intervalar na média de acerto dos grupos foi comparado por meio das análises do teste $t$ das amostras independentes. Não foi encontrada diferença na pontuação média para nenhuma das variáveis, embora as crianças com dislexia obtivessem menor pontuação em SP a diferença não foi significativa. Os resultados estão descritos na Tabela 2.

\section{Discussão}

Estudos têm mostrado dificuldades de disléxicos em tarefas de discriminação temporal dos sons em rápida alternância (Tallal \& Pierey, 1975) e manipulação fonológica (McAnally \& cols., 1997, Snowling \& Stackhouse, 2004). Ramus e cols. (2003) avaliaram adultos disléxicos em diferentes domínios e encontraram déficit 
fonológico em todos os disléxicos, mas aproximadamente $60 \%$ também apresentaram déficit no processamento auditivo.

No presente estudo, crianças disléxicas e com habilidades normais de leitura foram comparadas na capacidade de discriminar sons de sílabas com sonoridade similar e ponto de articulação diferente. De acordo com Russo e Behlau (1993), o ponto e o modo de produção são uns dos fatores que definem a identidade acústica do som e, de acordo com Serniclaes e colaboradores (2004a), a percepção das categorias fonológicas depende da extração dos traços acústicos, transformação analógica dos mesmos para as categorias fônicas e agrupamento para as categorias fonológicas. De fato, quando se considera o ponto e modo de articulação, os resultados deste estudo mostram que o número de acertos dos dois grupos foi menor nas sílabas sonoras (SS) do que nas SP e SI. Esta diferença pode ser explicada pelo fato das SS oferecerem menos dicas fonológicas aos sujeitos e as sílabas SP terem identidade sonora definida pelo diferente ponto de articulação.

Resultado similar foi encontrado por Capovilla e Capovilla (2002) em crianças brasileiras de uma escola regular da $1^{\mathrm{a}}$ a $4^{\mathrm{a}}$ série. De modo geral, bons e maus leitores do ensino fundamental apresentam número menor de acertos em sílabas que diferem quanto à sonorização quando comparado com sílabas do diferem com relação ao ponto de articulação. Os autores encontraram interação entre média de acerto e o nível de leitura das crianças. Entre as crianças de sala regular, a proporção de acerto foi menor para aquelas que tiveram desempenho pior nas provas de leitura sendo, portanto, consideradas pelos autores como maus leitores.

A análise dos resultados, em função dos IEES, mostrou que os disléxicos apresentaram média de acerto menor para todos os tipos de sílabas, mas a significância foi encontrada somente para sílabas SP e SS com IEEs longos. Desta maneira, o déficit de percepção e discriminação sonora observado em disléxicos pode ocorrer em alguns dos níveis: acústico, fônico ou fonológico. De maneira similar, Capovilla e Capovilla (2002) encontraram interação entre o número de acertos e os IEEs em escolares bons e maus leitores. A curva de acerto manteve-se crescente até 2,5 segundos e passou à decrescer nos IEEs maiores. Esse padrão foi mais evidente em grupo dos maus leitores sendo a diferença maior nos tempos extremos em relação aos bons leitores. Essa diferença foi interpretada, de acordo com os autores, como uma dificuldade dos maus leitores na velocidade de processamento em intervalos curtos e prejuízo na memória de trabalho fonológica em intervalos longos.

No presente estudo, um padrão diferente foi encontrado para cada categoria de sílabas em função do IEE. A média de acerto foi alta nas sílabas SI e SP no intervalo curto, mas para as sílabas SS, a média de acerto foi baixa. À medida que o IEE aumentou, o número médio de acertos declinou da seguinte forma: para ambos os grupos nas sílabas SI, mas apenas para os disléxicos nas sílabas SP. Já para as sílabas SS foi observada uma função crescente ao longo dos IEEs, sendo observado ganho menor para os disléxicos, quando comparados com os controles nos IEEs longos. Além disso, em sílabas SS (/fa/-/va/ e $/ \mathrm{ka} /-/ \mathrm{ga} /)$ e em sílabas SP (/za/-/va/), os disléxicos acertaram menos, o que pode indicar uma dificuldade específica de discriminação para esses estímulos. A discussão dos resultados a partir da perspectiva da percepção categórica das sílabas e do funcionamento da memória de trabalho pode possibilitar uma maior compreensão dos processamentos envolvidos nos níveis: auditivo, fônico e fonológico. Assim, de acordo com Russo e Behlau (1993) a percepção de fala depende, entre outros fatores, da identificação de freqüência, intensidade e dimensões temporais dos sons articulados. Quanto aos aspectos fônicos das sílabas em que foram observadas diferenças entre os dois grupos as fricativas labiodentais /f/ e /v/ apresentam as menores intensidades sonoras, quando comparadas com outras consoantes do português do Brasil. Já as consoantes plosivas velares / k, g/ apresentam intensidade forte e freqüência intermediária de 1.500 a $4.000 \mathrm{~Hz}$. Por último a consoante fricativa $/ z /$ é formada por som forte e agudo com uma faixa de freqüência entre 4.500 a $8.000 \mathrm{~Hz}$

Moody, Studdenrt-Kennedy e Brady (1997) estudaram a capacidade de discriminar silabas em rápida alternância em bons e maus leitores. A discriminação foi avaliada em sílabas foneticamente similares /ba/-/da/ e dissimilares /da/-/sa/. Somente em pares similares foi encontrada diferença no desempenho entre os grupos. Serniclaes e colaboradores (2004b) argumentam que as diferença na discriminação e categorização das sílabas é comumente encontrada quando, por exemplo, é satisfeita a seguinte condição: categorias silábicas não representam uma dificuldade discriminatória nem muito baixa e 
nem alta para os controles. Neste caso, aparece o déficit dos participantes disléxicos na discriminação de categorias diferentes, mas não de categorias iguais. Tal característica foi observada no presente estudo, em sílabas SS e SP, e na ausência de diferença em sílabas SI. Este padrão pode ser atribuído à má formação das fronteiras fonológicas durante o desenvolvimento e preservação por parte dos disléxicos do julgamento alofônico. Durante o julgamento alofônico, na falta de representação fonética específica para cada som, o sistema perceptual dependeria do julgamento das variações sonoras dentro de um grupo fonético (Serniclaes \& cols., 2004b). Em suma, os resultados encontrados neste estudo são coerentes com achados do Serniclaes e colaboradores (2004a e 2004b), uma vez que nos pares consonantais /v/-/f/ e / k/-/g/ a pequena variação sonora é determinada pelo VOT. A formação das fronteiras fonológicas depende da correta percepção do VOT ao longo da especialização lingüística da língua materna das crianças na primeira infância (Serniclaes \& cols., 2004b).

A realização das tarefas usadas no presente estudo demanda utilização da memória de trabalho pois, para dar uma resposta correta o participante deveria manter na memória o primeiro estímulo apresentado a fim de comparar com o estímulo subseqüente apresentado após um intervalo de tempo variado. O Efeito de Similaridade Fonológica (ESF) proposto por Repovs e Baddeley (2006) foi observada no presente estudo nas provas de reconhecimento dos sons em intervalos curtos nas sílabas com sonoridade similar SS. Assim, o maior número de respostas incorretas observadas nas sílabas SS pode ser explicado em função da interferência do ESF. Já nos pares de sílabas SP que variavam principalmente com relação ao ponto de articulação, não foi observada interferência do ESF, pois os participantes obtiveram número maior de acertos.

Outro resultado importante do presente estudo foi a observação do aumento do número de acerto com aumento do IEEs. Como o reconhecimento dos estímulos é facilitado pela formação das categorias sonoras (Luo \& cols., 2005), pode-se inferir que o aumento do intervalo entre os estímulos aumente também a seletividade categórica para os sons próximos e, conseqüentemente, aumente o número de respostas corretas. Os resultados do presente estudo mostram que os disléxicos acertam menos que os controles no julgamento de estímulos de categorias diferentes pelo ponto de articulação à medida que os IEEs aumentam para mais de 10 segundos. Estes achados corroboram a hipótese de uma dificuldade de discriminação de sons distintos, levando a suposição de que estas categorias de sons são bem menos definidas para os disléxicos do que para os controles.

O bom desempenho nas provas usadas neste estudo dependeu da correta comparação do segundo estímulo com o estímulo previamente armazenado. Assim, as dificuldades observadas nos disléxicos envolvem, pelo menos, dois componentes que se inter-relacionam e devem ser mais bem compreendidos na dislexia: discriminação de sons e memória de trabalho. Pelo menos 3 questões devem ser respondidas em estudos futuros para a compreensão das dificuldades específicas da dislexia relacionadas com memória de trabalho e processamento fonológico: 1) como se dá o armazenamento do primeiro estímulo apresentado; 2) qual é qualidade da representação sonora do primeiro estímulo depois de alguns segundos; 3) como o segundo estímulo apresentado interfere na representação sonora do primeiro estímulo.

O presente estudo avaliou a capacidade de discriminação de sílabas pareadas por similaridade sonora e ponto de articulação em crianças disléxicas e bons leitores. Resultados mostram que disléxicos cometem mais erros no julgamento de sílabas com similaridade sonora, que parece estar diretamente relacionado com déficit no processamento fonológico e na memória fonológica. A utilização de provas de julgamento de prova com par SOM/SOM discriminou melhor os disléxicos dos controles do que as provas SOM/ESCRITA.

\section{Referências}

Ackermann, H. \& Hertrich, I. (1997). Voice onset time in ataxic dysarthria. Brain and Language, 56, 321-333.

Bishop, D. V. M., Carlyon, R. P., Deeks, J. M. \& Bishop, S. (1999). Auditory temporal processing impairment: neither necessary nor sufficient for causing language impairment in children. Journal of Speech, Language, and Hearing Research, 42, 1295-1310.

Capovilla, F. C. \& Capovilla, A. G. S. (2002). Atraso na aquisição de leitura: Relação com problemas de discriminação fonológica, 
velocidade de processamento e memória fonológica. Em Capovilla, F. C (Org.) Neuropsicologia e aprendizagem. São Paulo, SP: SNBp. p. 213-317.

Kibby, M. Y., Marks, W., Morgan, S. \& Long, C. J. (2004). Specific impairment in developmental reading disabilities: a working memory approach. J Learn Disabil., 37 (4), 349-63.

Luo, H., Husain, F. T., Horowitz, B. \& Poeppel, D. (2005). Discrimination and categorization of speech and non-speech sounds in an MEG delayed-match-to-sample study. NeuroImage, 28, 59-71.

Lyon, G. R. (2003). Defining dyslexia, comorbidity, teachers' knowledge of language and reading. Annals of Dyslexia. 53,1-14.

Macedo, E. C., Capovilla, F. C., Nikaedo, C. C., Orsati, F. T., Lukasova, K., Capovilla, A. G. S. \& Diana, C. (2005). Teleavaliação da habilidade de leitura no Ensino Infantil e Fundamental. Psicologia Escolar e Educacional, 9(1), 127-134.

McAnally, K. I., Hansen, P. C., Cornelissen, P. L. \& Stein, J. F. (1997). Effect of time and frequency manipulation on syllable perception in developmental dyslexics. J. Exp. Child Psychol, 66, 211-235.

Moody, M., Studdenrt-Kennedy, M. \& Brady, S. (1997). Speech perception deficits in poor readers: auditory processing or phonological coding? J. Exp. Child Psychol, 64, 199-231.

Ramus, F., Rosen, S., Dakin, S. C., Day, B. L., Castellote, J. M., White, S. \& Frith, U. (2003).
Theories of developmental dyslexia: insights from a multiple case study of dyslexic adults. Brain, 126, 841-865.

Repovs, G. \& Baddeley, A. (2006). The multicomponent model of working memory: explorations in experimental cognitive psychology. Neuroscience, 139, 5-21.

Russo, I. \& Behlau, M. (1993). Percepção da Fala: Análise Acústica. São Paulo, SP: Editora Lovise.

Sernicales, W., Van Heghe, S., Mousty, P., Carré, R. \& Sprenger-Charolles L (2004a) Allophonic mode of speech perception in dyslexia. $J$ Exp Child Psychol, 87(4), 336-61.

Serniclaes, W., Ventura, P., Morais, J. \& Kolinsky, R. (2004b). Categorical perception of speech sounds in illiterate adults. Cognition, 98, B35B44.

Snowling, M. J. \& Stackhouse, J. (2004). Dislexia, fala e linguagem: um manual do profissional. São Paulo, SP: Artmed.

Tallal, P. \& Pierey, M. (1975). Developmental aphasia: the perception of brief vovels and extended stop consonants. Neuropsychologia, 13, 69-74.

Wechsler, D. \& Figueredo, V. L. M. (2002). WISCIII: escala de inteligência Wechsler para crianças adaptação brasileira da $3^{a}$ edição. São Paulo, SP: Casa do Psicólogo.

Recebido em Abril de 2008

Reformulado em Outubro de 2008 Aprovado em Dezembro de 2008

Sobre os autores:

Katerina Lukasova é psicóloga e mestre em Distúrbios do Desenvolvimento pela Universidade Presbiteriana Mackenzie. Atua como professora do curso de Psicologia na Universidade Cruzeiro do Sul.

Elizeu Coutinho de Macedo é psicólogo, mestre e doutor em Psicologia Experimental pela Universidade de São Paulo. Atua como professor do programa de pós-graduação em Distúrbios do Desenvolvimento da Universidade Presbiteriana Mackenzie.

Anna Carolina Cassiano Barbosa é psicóloga e mestre em Distúrbios do Desenvolvimento pela Universidade Presbiteriana Mackenzie. 